\title{
COHOMOLOGY WITH EXOTIC SUPPORTS AND THE GENERALIZED EXCISION
}

\author{
SATYA DEO, DALIP SINGH JAMWAL and RAM KRISHAN
}

(Received 18 November 1986)

Communicated by J. H. Rubinstein

\begin{abstract}
The main result proved in this paper is the following. Suppose $X_{1}, X_{2}$ are two subspaces of a space $X$ such that $X=\operatorname{Int}\left(X_{1}\right) \cup X_{2}=X_{1} \cup \operatorname{Int}\left(X_{2}\right)$. Then the pair $\left(X_{1}, X_{2}\right)$ is a $\varphi$-excisive pair in sheaf cohomology for any family of supports $\varphi$ on $X$. This result settles an open question and includes all known results on $\varphi$-excisiveness in sheaf cohomology as its special cases. We construct several examples to illustrate our main theorem and to show that it is, in fact, quite sharp.

1980 Mathematics subject classification (Amer. Math. Soc.) (1985 Revision): primary 55 N 30; secondary $55 \mathrm{~N} 40$.
\end{abstract}

\section{Introduction}

Let $\varphi$ be a family of supports on a given topological space $X$. Recall that a pair $\left(X_{1}, X_{2}\right)$ of subspaces of $X$ is said to be $\varphi$-excisive if the inclusion map $\left(X_{1}, X_{1} \cap X_{2}\right) \rightarrow\left(X_{1} \cup X_{2}, X_{2}\right)$ induces isomorphism

$$
H_{\varphi \cap\left(X_{1} \cup X_{2}\right)}^{*}\left(X_{1} \cup X_{2}, X_{2} ; \mathscr{A}\right) \rightarrow H_{\varphi \cap X_{1}}^{*}\left(X_{1}, X_{1} \cap X_{2} ; \mathscr{A}\right)
$$

in sheaf cohomology for any sheaf $\mathscr{A}$ of $R$-modules on $X$. As usual this implies that the Mayer-Vietoris sequence of sheaf cohomology for the pair $\left(X_{1}, X_{2}\right)$, with respect to any sheaf $\mathscr{A}$ on $X$ and with supports in $\varphi$, is exact. The results on $\varphi$-excisiveness stated in the following theorem are well known ([1], page 68).

(C) 1988 Australian Mathematical Society $0263-6115 / 88 \$ A 2.00+0.00$ 
THEOREM. A pair $\left(X_{1}, X_{2}\right)$ of subspaces of a space $X$ is $\varphi$-excisive for a given family $\varphi$ of supports on $X$ if any one of the following conditions is satisfied:

(1.1) $X=\operatorname{Int}\left(X_{1}\right) \cup \operatorname{Int}\left(X_{2}\right)$.

(1.2) $X=X_{1} \cup X_{2}$ and both $X_{1}, X_{2}$ are closed in $X$.

(1.3) $X=\operatorname{Int}\left(X_{1}\right) \cup X_{2}=X_{1} \cup \operatorname{Int}\left(X_{2}\right)$ and $X_{1}, X_{2}, X_{1} \cap X_{2}$ are all $\varphi$-taut in $X$.

The result (1.3) above, discovered in the context of sheaf cohomology, is relatively new, and as pointed out in [2], substantially enlarges the class of excisive pairs (and hence the validity of Mayer-Vietoris exact sequences) for sheaf cohomology as well as for Alexander-Spanier cohomology.

The first attempt to answer this question was made in [2] where the first author has shown that under a hypothesis which is only slightly weaker than the condition $X=\operatorname{Int}\left(X_{1}\right) \cup X_{2}=X_{1} \cup \operatorname{Int}\left(X_{2}\right)$, the tautness conditions stated in (1.3) are really needed. The main question, however, remained unresolved.

The basic objective of this paper is to answer the above question completely. We prove (Theorem 2.1) that the tautness conditions in (1.3) are superfluous. Our proof exploits the so called second resolution of a sheaf due to Godement. The usual known methods of proving similar or weaker results do not seem to generalize to this case. It must be pointed out here that this is yet another example of a situation where Godement's second resolution works so nicely and others do not seem to be even applicable. Note that results (1.1) and (1.2) are now special cases of our Theorem 2.1 and hence our main result not only generalizes all of the previously known results on $\varphi$-excisiveness, but also gives a unified proof of all of them. We also show that Theorem 2.1 is quite sharp. This is done by constructing an example (Example 4.3) of a pair $\left(X_{1}, X_{2}\right)$ of subspaces of a space $X$ such that $X=\operatorname{Int}\left(X_{1}\right) \cup X_{2}$ and $X_{1}, X_{2}, X_{1} \cap X_{2}$ are all $\varphi$-taut in $X$, but even then the pair $\left(X_{1}, X_{2}\right)$ is not $\varphi$-excisive. Finally we go on to illustrate our theorem by constructing subspaces $X_{1}, X_{2}$ of a space $X$ and a family $\varphi$ of supports on $X$ such that $X=\operatorname{Int}\left(X_{1}\right) \cup X_{2}=X_{1} \cup \operatorname{Int}\left(X_{2}\right)$ and $X_{1}, X_{2}, X_{1} \cap X_{2}$ are not $\varphi$-taut, but the Mayer-Vietoris sequence of the pair $\left(X_{1}, X_{2}\right)$ for the sheaf cohomology of a sheaf on $X$ with respect to $\varphi$ is exact (Example 4.4). The construction of all these examples leads us to study the sheaf cohomology of some contractible spaces with exotic supports. These are developed in Section 3. Various computations of sheaf cohomology with these exotic supports turn out to be quite interesting and we have described several situations which seem to indicate that they may have potential applications in some other contexts also.

Unless stated otherwise, we have used the constant sheaf $\mathbf{Z}$ of integers throughout the paper. For brevity $H_{\varphi}^{*}(X ; \mathbf{Z})$ has been denoted by only $H_{\varphi}^{*}(X)$. For a family $\varphi$ of supports, the extent $E(\varphi)$ of $\varphi$ is the union of all members of $\varphi$. If 
$A \subset X$, then $H_{\varphi \cap A}^{*}(A)$ has been denoted by only $H_{\varphi}^{*}(A)$. For all other definitions, notations and results we refer to [1].

\section{Main result-the general excision theorem}

In this section we propose to prove the following exicision theoem for sheaf cohomology which generalizes all the known results on excisive pairs and also settles an open question in this area (see the introduction).

THEOREM 2.1. Let $X$ be a topological space and $X_{1}, X_{2}$ be two subspaces of $X$ such that $X=\operatorname{Int}\left(X_{1}\right) \cup X_{2}=X_{1} \cup \operatorname{Int}\left(X_{2}\right)$. Then the pair $\left(X_{1}, X_{2}\right)$ is $\varphi$-excisive for any family $\varphi$ of supports on $X$.

Before proving the result we must point out that the two equations $X=$ $\operatorname{Int}\left(X_{1}\right) \cup X_{2}=X_{1} \cup \operatorname{Int}\left(X_{2}\right)$ are clearly equivalent to the single equation $X=$ $\operatorname{Int}\left(X_{1}\right) \cup \operatorname{Int}\left(X_{2}\right) \cup\left(X_{1} \cap X_{2}\right)$. This remark has been implicitly used in the proof.

Let $\mathscr{A}$ be any sheaf of $R$-modules on $X$. As stated in the introduction, we shall use the second canonical resolution $\mathscr{F}^{*}(X ; \mathscr{A})$ of $\mathscr{A}$ (due to Godement [5]) in the proof of the above theorem (see [1], page 28 for details). Let $F_{\varphi}^{p}(X ; \mathscr{A})=$ $\Gamma_{\varphi} \mathscr{F}^{p}(X ; \mathscr{A})$. Then for any family of supports $\varphi$ on $X$, the cohomology of the cochain complex

$$
0 \rightarrow F_{\varphi}^{0}(X: \mathscr{A}) \rightarrow F_{\varphi}^{1}(X ; \mathscr{A}) \rightarrow \cdots \rightarrow F_{\varphi}^{p}(X ; \mathscr{A}) \rightarrow \cdots
$$

gives the sheaf cohomology $H_{\varphi}^{*}(X ; \mathscr{A})$ of $X$ with coefficients in $\mathscr{A}$ and supports in $\varphi$. Recall that the cochain groups $F^{p}(X ; \mathscr{A})$ can be defined (analogous to Alexander-Spanier cochain complex) as a quotient of modules $M^{p}(X ; \mathscr{A})$, here $M^{p}(X ; \mathscr{A})$ denote the module of all functions $f: X^{p+1} \rightarrow \mathscr{A}$ such that $f\left(x_{0}, \ldots, x_{p}\right) \in \mathscr{A}_{x_{p}}$ with pointwise operations. The surjective homomorphism $\psi_{p}: M^{p}(X ; \mathscr{A}) \rightarrow F^{p}(X ; \mathscr{A})$ defining $F^{p}(X ; \mathscr{A})$ as the quotient has the following elements in its kernel [ibid] : $f \in \operatorname{Ker} \psi_{p}$ if for each $(q+1)$-tuple $\left(x_{0}, \ldots, x_{q}\right)$ $(q=0, \ldots, p-1)$ there is an open neighbourhood $U\left(x_{0}, \ldots, x_{q}\right)$ of $x_{q}$ in $X$ such that if

$$
x_{1} \in U\left(x_{0}\right), x_{2} \in U\left(x_{0}, x_{1}\right), \ldots, x_{p} \in U\left(x_{0}, \ldots, x_{p-1}\right),
$$

then $f\left(x_{0}, \ldots, x_{p}\right)=0$. Now for any subspace $A$ of $X$, let $M^{p}(X, A ; \mathscr{A})$ denote the set of all those $f \in M^{p}(X ; \mathscr{A})$ such that $f\left(x_{0}, \ldots, x_{p}\right)=0$ if $x_{i} \in A$ for each $i=0, \ldots, p$. If $F^{p}(X, A ; \mathscr{A})$ denotes the module $\psi_{p}\left(M^{p}(X, A ; \mathscr{A})\right)$, then it is known ([ibid], page 64) that the cochain complex $F^{*}(X, A ; \mathscr{A})$ defines the relative cohomology groups $H^{*}(X, A ; \mathscr{A})$ of the pair $(X, A)$. Now let $\left(X_{1}, X_{2}\right)$ be any pair of subspaces of $X$. Define $M^{p}\left(X, X_{1}, X_{2} ; \mathscr{A}\right)=M^{p}\left(X, X_{1} ; \mathscr{A}\right) \cap$ $M^{p}\left(X, X_{2} ; \mathscr{A}\right)$ and let $F^{p}\left(X, X_{1}, X_{2} ; \mathscr{A}\right)=\psi_{p}\left(M^{p}\left(X, X_{1}, X_{2} ; \mathscr{A}\right)\right)$. Then the 
presheaf $U \rightarrow F^{p}\left(U, U \cap X_{1}, U \cap X_{2} ; \mathscr{A}\right)$ defines a flabby sheaf $\mathscr{F}^{p}\left(X, X_{1}, X_{2} ; \mathscr{A}\right)$ as the kernel of the surjective homomorphism

$$
\mathscr{F}^{p}\left(X, X_{1} ; \mathscr{A}\right) \rightarrow \mathscr{F}^{p}\left(X_{2}, X_{1} \cap X_{2} ; \mathscr{A}\right) .
$$

If we put $\Gamma_{\varphi} \mathscr{F}^{p}\left(X, X_{1}, X_{2} ; \mathscr{A}\right)=F_{\varphi}^{p}\left(X, X_{1}, X_{2} ; \mathscr{A}\right)$ etc., then it is a consequence of $3 \times 3$ lemma and relative cohomology of various pairs that the sequence

$$
0 \rightarrow F_{\varphi}^{*}\left(X, X_{1}, X_{2} ; \mathscr{A}\right) \rightarrow F_{\varphi}^{*}\left(X, X_{1} ; \mathscr{A}\right) \rightarrow F_{\varphi \cap X_{2}}^{*}\left(X_{2}, X_{1} \cap X_{2} ; \mathscr{A}\right) \rightarrow 0
$$

of cochain complexes is exact. Hence the pair $\left(X_{1}, X_{2}\right)$, where $X=X_{1} \cup X_{2}$ is $\varphi$-excisive, if and only if $H_{\varphi}^{p}\left(X_{1} \cup X_{2}, X_{1}, X_{2} ; \mathscr{A}\right)=0$ for each $p \geq 0$ [ibid, page 67].

PROOF OF THE THEOREM. With the given hypothesis of the theorem viz, $X=\operatorname{Int}\left(X_{1}\right) \cup X_{2}=X_{1} \cup \operatorname{Int}\left(X_{2}\right)$ we shall show that the sheaf $\mathscr{F}^{*}\left(X, X_{1}, X_{2} ; \mathscr{A}\right)$ on $X$ itself is a zero sheaf for any sheaf $\mathscr{A}$ on $X$. Then it will follow from the above discussion that $\left(X_{1}, X_{2}\right)$ is $\varphi$-excisive for any $\varphi$. To prove that $\mathscr{F}^{p}\left(X, X_{1}, X_{2} ; \mathscr{A}\right)=0$, it is clearly enough to prove the following: If $f \in$ $M^{p}\left(X, X_{1} ; \mathscr{A}\right) \cap M^{p}\left(X, X_{2} ; \mathscr{A}\right)$, then $f \in \operatorname{Ker} \psi_{p}$; here $\psi_{p}$ is the homomorphism defined above. Now we proceed to prove this contention.

Let $B=X-\left(\operatorname{Int} X_{1} \cup \operatorname{Int} X_{2}\right)$. Then $B$ is a closed set of $X$ contained in $X_{1} \cap X_{2}$. (If $B=\varnothing$ our proof yields the proof of Theorem (1.1). Theorem (1.2) is clearly a special case of our Theorem.) Let $V=\operatorname{Int}\left(X_{1}\right) \cap \operatorname{Int}\left(X_{2}\right)$. Then $V$ is an open set of $X$ contained in $X_{1} \cap X_{2}$. For each $(q+1)$-tuple $\left(x_{0}, \ldots, x_{q}\right)$ we will define an open neighbourhood $U\left(x_{0}, \ldots, x_{q}\right)$ of $x_{q}$ in $X$ by induction $(q+1 \leq p-1)$. For $q=0$, we define

$$
U\left(x_{0}\right)= \begin{cases}X & \text { if } x_{0} \in B \\ \operatorname{Int}\left(X_{1}\right) & \text { if } x_{0} \in \operatorname{Int}\left(X_{1}\right)-V \\ \operatorname{Int}\left(X_{2}\right) & \text { if } x_{0} \in \operatorname{Int}\left(X_{2}\right)-V \\ V & \text { if } x_{0} \in V .\end{cases}
$$

Now suppose $U\left(x_{0}, \ldots, x_{q}\right)$ have been defined (for $q+1<p-1$ ) and have the following properties.

(a) $U\left(x_{0}, \ldots, x_{q}\right)=X$ implies $x_{q} \in B$,

(b) $U\left(x_{0}, \ldots, x_{q}\right)=\operatorname{Int}\left(X_{1}\right)$ implies $x_{q} \in \operatorname{Int}\left(X_{1}\right)-V$,

(c) $U\left(x_{0}, \ldots, x_{q}\right)=\operatorname{Int}\left(X_{2}\right)$ implies $x_{q} \in \operatorname{Int}\left(X_{2}\right)-V$, and

(d) $U\left(x_{0}, \ldots, x_{q}\right)=V$ implies $x_{q} \in V$.

Then we define $U\left(x_{0}, \ldots, x_{q+1}\right)$ as follows.

$\left(\mathrm{a}^{\prime}\right)$ If $U\left(x_{0}, \cdots, x_{q}\right)=X$, then $x_{q} \in B$ and we define

$$
U\left(x_{0}, \ldots, x_{q+1}\right)= \begin{cases}X & \text { if } x_{q+1} \in B, \\ \operatorname{Int}\left(X_{1}\right) & \text { if } x_{q+1} \in \operatorname{Int}\left(X_{1}\right)-V \\ \operatorname{Int}\left(X_{2}\right) & \text { if } x_{q+1} \in \operatorname{Int}\left(X_{2}\right)-V \\ V & \text { if } x_{q+1} \in V .\end{cases}
$$


$\left(\mathrm{b}^{\prime}\right)$ If $U\left(x_{0}, \ldots, x_{q}\right)=\operatorname{Int}\left(X_{1}\right)$, then $x_{q} \in \operatorname{Int}\left(X_{1}\right)-V$ and we define

$$
U\left(x_{0}, \ldots, x_{q+1}\right)= \begin{cases}\operatorname{Int}\left(X_{1}\right) & \text { if } x_{q+1} \in \operatorname{Int}\left(X_{1}\right)-V, \\ V & \text { if } x_{q+1} \in V .\end{cases}
$$

$\left(c^{\prime}\right)$ If $U\left(x_{0}, \ldots, x_{q}\right)=\operatorname{Int}\left(X_{2}\right)$, then $x_{q} \in \operatorname{Int}\left(X_{2}\right)-V$ and we define

$$
U\left(x_{0}, \ldots, x_{q+1}\right)= \begin{cases}\operatorname{Int}\left(X_{2}\right) & \text { if } x_{q+1} \in \operatorname{Int}\left(X_{2}\right)-V, \\ V & \text { if } x_{q+1} \in V .\end{cases}
$$

(d') If $U\left(x_{0}, \ldots, x_{q}\right)=V$, then $x_{q} \in V$ and we define

$$
U\left(x_{0}, \ldots, x_{q+1}\right)=V \quad \text { for every } x_{q+1} \in V .
$$

Thus $U\left(x_{0}, \ldots, x_{q+1}\right)$ have also been defined and satisfy the inductive hypothesis. Therefore, by induction $U\left(x_{0}, \ldots, x_{q}\right)$ have been defined for all $q$ such that $q+1 \leq$ $p-1$. Now if

$$
x_{1} \in U\left(X_{0}\right), x_{2} \in U\left(x_{0}, x_{1}\right), \ldots, x_{p} \in U\left(x_{0}, \ldots, x_{p-1}\right),
$$

then it is easy to see that $x_{0}, x_{1}, \ldots, x_{p}$ belong (respectively) to a decreasing sequence $A_{0} \supseteq A_{1} \supseteq \cdots \supseteq A_{p-1}$ of subsets of $X$ where $A_{i}=B, \operatorname{Int}\left(X_{1}\right), \operatorname{Int}\left(X_{2}\right)$ or $V$ for each $i=0,1, \ldots, p-1$. In any case $x_{0}, \ldots, x_{p} \in X_{1}$ or $x_{0}, \ldots, x_{p} \in X_{2}$ and so $f\left(x_{0}, \ldots, x_{p}\right)=0$. This proves our assertion and that completes the proof of our theorem.

Next let us recall the definition of Alexander-Spanier cohomology of a topological pair $(X, A)$ with coefficients in a $R$-module $G$. This turns out to be the cohomology of the cochain complex $C^{*}(X, A) / C_{0}^{*}(X)$ where $C^{p}(X, A)$ denotes the $R$-module of all those functions $\alpha: X^{p+1} \rightarrow G$ which are locally zero on $A$, and $C_{0}^{p}(X)$ is the $R$-module of all functions $\beta: X^{p+1} \rightarrow G$ which are locally zero on $X([6]$, page 309). Just as in the proof of the above theorem one can rather easily modify the arguments and prove the following:

If $X=\operatorname{Int}\left(X_{1}\right) \cup X_{2}=X_{1} \cup \operatorname{Int}\left(X_{2}\right)$ and $\alpha: X^{p+1} \rightarrow G$ is locally zero on $X_{1}$ as well as locally zero on $X_{2}$, then $\alpha$ is locally zero on $X$. To see this suppose $\mathscr{U}$ is an open covering of $X_{1}$ and $\mathscr{V}$ is an open covering of $X_{2}$ (members of $\mathscr{U}$ and $\mathscr{V}$ are open in $X$ ) such that $\alpha$ is zero on members of $\mathscr{U} \cap X_{1}$ as well as on $\mathscr{V} \cap X_{2}$. Then one can easily see that $\alpha$ is zero on each member of the open covering $\left(\mathscr{U} \cap \operatorname{Int}\left(X_{1}\right)\right) \cup\left(\mathscr{V} \cap \operatorname{Int}\left(X_{2}\right)\right) \cup(\mathscr{U} \cap \mathscr{V})$ of $X$. This implies that the kernel of the onto cochain map

$$
C^{*}\left(X, X_{1}\right) \rightarrow C^{*}\left(X_{2}, X_{1} \cap X_{2}\right)
$$

consists of only locally zero functions on $X$ and so by passing to the quotients (by $C_{0}^{*}(X)$ ), we obtain that the inclusion map $\left(X_{2}, X_{1} \cap X_{2}\right) \rightarrow\left(X, X_{1}\right)$ induces isomorphism in the Alexander-Spanier cochain complexes $\bar{C}^{*}\left(X, X_{1}\right) \rightarrow$ $\bar{C}^{*}\left(X_{2}, X_{1} \cap X_{2}\right)$. Hence it induces isomorphism in cohomology. This prove the following corollary which generalizes the known results on excisive couples for Alexander-Spanier cohomology. 
COROLlARY 2.2. If $X_{1}, X_{2}$ are two subspaces of a space $X$ such that $X=$ $\operatorname{Int}\left(X_{1}\right) \cup X_{2}=X_{1} \cup \operatorname{Int}\left(X_{2}\right)$, then $\left(X_{1}, X_{2}\right)$ is an excisive couple for the Alexander-Spanier cohomology.

REMARK 2.3. It can easily be verified that Theorem 2.1 (respectively Corollary 2.2) is equivalent to the following General Excision Theorem for sheaf cohomology (respectively Alexander-Spanier cohomology). Let $(X, A)$ be a topological pair and $B$ be a subset of $X$ such that $B \subset \operatorname{Int} A$ and $\bar{B} \subset A$, then the subset $B$ can be excised from the pair $(X, A)$. In particular, if $A$ is closed then $\operatorname{Int} A$ can always be excised for each of these cohomology theories.

\section{Cohomology groups with exotic supports}

Let $X, Y$ be two spaces with the family of supports $\varphi$ and $\psi$ repsectively. A map $f: X \rightarrow Y$ is said to be proper (w.r.t. $\varphi$ and $\psi$ ) if $f^{-1}(K) \in \varphi$ for each $K \in \Psi$. It is a well known fact that if $L$ is a constant sheaf, then any two properly homotopic maps induce identical homomorphisms from $H_{\psi}^{*}(Y, L) \rightarrow$ $H_{\varphi}^{*}(X, L)$ ([1], Theorem 11.4, page 56), that is, homotopy axiom holds for the sheaf cohomology of constant sheaves. In this section we are going to make use of this homotopy axiom and the exactness of Mayer-Vietoris sequence in sheaf cohomology to perform some interesting computations of cohomology groups of some contractible spaces with exotic supports. Let us consider the following situation:

Suppose $\varphi$ is a family of supports on a space $X$. For any subset $A$ of $X$, the inclusion map $A \rightarrow X$ is clearly proper with respect to $\varphi \cap A$ and $\varphi$. Suppose $A$ consists of a single point of $X$. Then the constant map $c: X \rightarrow A$, however, need not be proper. But if $\varphi \cap A$ consists of only the null set, then obviously this constant map is also proper. Identity map $I_{X}: X \rightarrow X$ is always proper. In case $X$ is contractible, we want to know under what conditions the identity map $I_{X}$ and the constant map $c: X \rightarrow X$ (which are proper) are also properly homotopic. We shall give an example later (Example 3.4(c)) to show that these proper maps need not be properly homotopic. If a family $\varphi$ of supports contains only the null set we shall refer it as the "empty support"; if it contains a singleton set and the null set then we shall refer it as a "point support" family. A space $X$ will be said to be $\varphi$-contractible if the identity map of the space $X$ is properly homotopic to a constant map to one of its points. It follows from the homotopy axiom stated above that in case the point is not in $\operatorname{Ext}(\varphi)$, then $H_{\varphi}^{p}(X)=0$ for all $p$, but in case the point is in $\operatorname{Ext}(\varphi)$, then $H_{\varphi}^{p}(X)=0$ for all $p \neq 0$ and $H_{\varphi}^{0}(X ; \mathbf{Z}) \approx \mathbb{Z}$ (for coefficients in the constant sheaf $\mathbf{Z}$ of abelian groups).

First of all, let us prove the following 
Proposition 3.1. Let $E$ be any starlike (not necessarily open or closed) subset of $\mathbf{R}^{n}$ and $\varphi=$ cld $\mid \mathbf{R}^{n}-E$ be the family of all closed subsets of $\mathbf{R}^{n}$ which are contained in $\mathbf{R}^{n}-E$. Then $\mathbf{R}^{n}$ is $\varphi$-contractible to the star-centre $p$ of $E$.

ProOF. Star-centre of $E$ is the point $p$ of $E$ from which all other points of $E$ can be joined by a line segment lying in $E$. We shall show that the usual homotopy $F: \mathbf{R}^{n} \times I \rightarrow \mathbf{R}^{n}$ defined by $F(x, t)=(1-t) x+t p$ is a proper homotopy. It is only necessary to verify that if $K \in \varphi$, then $F^{-1}(K) \in \varphi \times I$. Since $F$ is continuous and $K$ is closed in $\mathbf{R}^{n}, F^{-1}(K)$ is obviously closed in $\mathbf{R}^{n} \times I$. Then only thing to be observed is that $F^{-1}(K) \subset K^{\prime} \times I$ where $K^{\prime}$ is the union of all infinite line segments starting from points of $K$ moving away from $p$. Thus $K^{\prime}$ is closed in $\mathbf{R}^{n}$, and by definition of $\varphi$, belongs to $\varphi$. Hence $F^{-1}(K) \in \varphi \times I$.

It is clear from the proof above that instead of $\mathbf{R}^{n}$ we can take any starlike subset $Y$ of $\mathrm{R}^{n}$ provided the star-centres of both $Y$ and $E$ are same or else we can choose such a centre. The whole point in obtaining a $\varphi$-contraction is that the support family must be such that whenever $Q \in \operatorname{Ext}(\varphi)$, then any point $Q^{\prime}$ with the property that $Q$ divides the line segment $P Q^{\prime}$ internally must also be in $\operatorname{Ext}(\varphi)$. In fact we have

COROLLARY 3.2. Let $E$ be any bounded convex subset of $\mathbf{R}^{n}$ and $\varphi$ be a family of supports on $E$. Suppose there is a point $p \in E-\operatorname{Ext}(\varphi)$ such that whenever $Q \in \operatorname{Ext}(\varphi)$, the produced portion $Q Q^{\prime}$ of the line segment $P Q^{\prime}$ through $Q$ must also be in $\operatorname{Ext}(\varphi)$. Then $E$ is $\varphi$-contractible to the point $P$.

As a special case it is apparent from the above corollary that if the family of supports $\varphi$ consists of subsets all contained in the boundary of a disc $D^{n}$, then $D^{n}$ is $\varphi$-contractible to any of its interior points. It follows from the homotopy axiom for constant sheaves that $H_{\varphi}^{p}\left(D^{n}\right)=0$, for every $p \geq 0$.

Now we are going to make use of the above corollary to mention some other examples which will be used later on.

EXAMPLE 3.3. Let $\varphi=$ cld $\mid K$ where $K$ is some closed convex subset of the unit interval $I=[0,1]$ containing exactly one of the end points. Then $I$ is $\varphi$-contractible to any point of $I-K$ and so $H_{\varphi}^{p}(I)=0$, for every $p \geq 0$.

We shall always bear in mind the special case when $\varphi$ is a point support.

EXAMPLE 3.4. Let $J=(0,1)$ be the open interval and $\varphi=\operatorname{cld} \mid K$ where $K$ is some closed convex subset of $\mathbf{R}$ contained in $(0,1)$ (for example, $\varphi$ is a point support). Suppose $x \in K$. Then because $(0, x]$ and $[x, 1)$ both are $\varphi$-contractible to any point of $(0, x]-K$ and $[x, 1)-K$ respectively, $H_{\varphi}^{*}((0, x])=0=H_{\varphi}^{*}([x, 1))$. Hence by Mayer-Vietoris exact sequence $H_{\varphi}^{1}(J) \approx H_{\varphi}^{0}(\{x\}) \approx \mathbf{Z}, H_{\varphi}^{p}(J)=0$, $p \neq 1$. 
(a) We note that a similar result holds if $\varphi=\operatorname{cld} \mid J$.

(b) If we take $I=[0,1]$ and $\varphi=\operatorname{cld} \mid K$ where $K$ is some closed convex subset of $\mathbf{R}$ contained in $\operatorname{Int}(I)$, then it follows that $H_{\varphi}^{1}(I) \approx \mathbf{Z}$ and $H_{\varphi}^{p}(I)=0$, for every $p \neq 1$.

(c) This example shows, by the way, that $J$ can not be $\varphi$-contractible to any of its interior points for $\varphi$ defined above.

Proposition 3.5. Let $D^{n}$ be an $n$-disc and $\operatorname{Int}\left(D^{n}\right) \subseteq E \subseteq D^{n}$. Suppose $K$ is another disc contained in $\operatorname{Int}\left(D^{n}\right)$ and $\varphi=\operatorname{cld} \mid K$. Then,

$$
H_{\varphi}^{p}(E) \approx \begin{cases}\mathbf{Z} & \text { if } p=n, \\ 0 & \text { otherwise. }\end{cases}
$$

A similar (well known) result holds when $\varphi=\operatorname{cld} \mid \operatorname{Int}\left(D^{n}\right)$ (that is, compact supports).

Proof. Since $\operatorname{Int}\left(D^{n}\right)$ is open in $E$, it is $\varphi$-taut in $E$ and so $H_{\varphi}^{*}\left(E, \operatorname{Int}\left(D^{n}\right)\right)$ $\approx H_{\varphi \mid E-\operatorname{Int}\left(D^{n}\right)}^{*}(E)=0$, that is, $H_{\varphi}^{*}(E) \approx H_{\varphi}^{*}\left(\operatorname{Int}\left(D^{n}\right)\right)$. We shall compute the case when $E=D^{n}$. Without loss of generality we can assume that $D^{n}$ is the standard $n$-simplex $\Delta^{n}$. When $n=1$, the result follows from Example 3.4(b). Let us assume the result to be true for all $k<n$. Suppose $v_{0}$ is a vertex of $\Delta^{n}$. We divide $\Delta^{n}$ into two closed subsets $A, B$ having non-empty interiors such that $A \cap B$ is a $(n-1)$-simplex with $v_{0}$ as vertex. Now if $a$ is any boundary point of $A$ disjoint from $A \cap B$ and $b$ is any boundary point of $B$ disjoint from $A \cap B$, then $A, B$ both are $\varphi$-contractible to points $a$ and $b$ respectively. Hence by Mayer-Vietoris exact sequence for the pair $(A, B)$ we have

$$
H_{\varphi}^{p}\left(D^{n}\right) \approx H_{\varphi}^{p}(A \cup B) \approx H_{\varphi}^{p-1}(A \cap B)=H_{\varphi}^{p-1}\left(\Delta^{n-1}\right) \approx \begin{cases}\mathbb{Z} & \text { if } p=n, \\ 0 & \text { otherwise }\end{cases}
$$

COROLLARY 3.6. Let $K$ be any compact dise in $\mathbf{R}^{n}$ and $\varphi=\operatorname{cld} \mid K$. Then

$$
H_{\varphi}^{p}\left(\mathbf{R}^{n}\right) \approx \begin{cases}\mathbf{Z} & \text { if } p=n \\ 0 & \text { otherwise. }\end{cases}
$$

COROLLARY 3.7. Let $\varphi$ be the family of supports consisting of all closed subsets of the union of $m$-disjoint compact discs $K_{1}, \ldots, K_{m}$. Then,

$$
H_{\varphi}^{p}\left(\mathbf{R}^{n}\right) \approx \begin{cases}\frac{\mathbf{Z} \oplus \mathbf{Z} \oplus \cdots \oplus \mathbf{Z}}{m-\text { copies }} & \text { if } p=n \\ 0 & \text { otherwise. }\end{cases}
$$

ProOF. Take $m$-disjoint open balls $B\left(K_{i}, r_{i}\right)$, each ball containing one disc $K_{i}(i=1,2, \ldots, m)$. Since $W=\bigcup B\left(K_{i}, r_{i}\right)$ is open in $\mathbf{R}^{n}$, it is $\varphi$-taut and so 
$H_{\varphi}^{p}\left(\boldsymbol{R}^{n}, W\right) \approx H_{\varphi \mid \mathbf{R}^{n}-W}^{p}\left(\mathbf{R}^{n}\right)=0$, for every $p \geq 0$. Hence $H_{\varphi}^{p}\left(\boldsymbol{R}^{n}\right) \approx H_{\varphi}^{p}(W)$, for every $p \geq 0$. Now by induction and Mayer-Vietoris exact sequence we obtain

$$
H_{\varphi}^{p}(W) \approx \bigoplus_{i=1}^{m} H_{\varphi}^{p}\left(B\left(K_{i}, r_{i}\right)\right) \approx \begin{cases}\frac{\mathbb{Z} \oplus \cdots \oplus \mathbb{Z}}{m-\text { copies }} & \text { if } p=n \\ 0 & \text { otherwise }\end{cases}
$$

Proposition 3.8. Let $D^{n}$ be an $n$-disc and $\operatorname{Int}\left(D^{n}\right) \subseteq E \subseteq D^{n}$. Suppose $\varphi$ is a family of supports on $D^{n}$ containing the family $\operatorname{cld} \mid \operatorname{Int}\left(D^{n}\right)$ and exactly one line segment joining a point of $\operatorname{Int}\left(D^{n}\right)$ with a boundary point of $D^{n}$. Then $H_{\varphi \cap E}^{*}(E)=0$.

Proof. Again since $\operatorname{Int}\left(D^{n}\right)$ is open in $E$, it is $\varphi$-taut in $E$ and so $H_{\varphi}^{*}\left(E, \operatorname{Int}\left(D^{n}\right)\right) \approx H_{\varphi \mid E-\operatorname{Int}\left(D^{n}\right)}^{*}(E)=0$, since $E$ is $\left(\varphi \mid E-\operatorname{Int}\left(D^{n}\right)\right)$-contractible. Hence $H_{\varphi \cap E}^{*}(E) \approx H_{\varphi \cap \operatorname{Int}\left(D^{n}\right)}^{*}\left(\operatorname{Int}\left(D^{n}\right)\right)$. Let $\psi$ be the family of supports containing $\varphi$ and all closed subsets of $D^{n}$ which are contained in the boundary of $D^{n}$. Then $\psi \cap \operatorname{Int}\left(D^{n}\right)=\varphi \cap \operatorname{Int}\left(D^{n}\right)$ and we shall compute $H_{\varphi}^{*}\left(D^{n}\right)$. Assume again that $D^{n}$ is the standard $n$-simplex $\Delta^{n}$ and the boundary point of $\Delta^{n}$ which is joined by a line segment (lying in $\varphi$ ) to a point of $\operatorname{Int}\left(\Delta^{n}\right)$ be assumed as a vertex $v_{0}$. If $n=1$, then the result follows by Example 3.3. Hence assume the result to be true for all $k<n$. As before, let $A, B$ be two closed subsets of $\Delta^{n}$ having non-empty interiors such that $\Delta^{n}=A \cup B, A \cap B$ is a $(n-1)$-simplex having $v_{0}$ as a vertex. Then it is clear that $A, B$ are $\psi$-contractible and hence $\psi$-acyclic and by induction hypothesis $A \cap B$ is also $\psi$-acyclic. Therefore, by Mayer-Vietoris sequence, $A \cup B$ is $\psi$-acyclic.

REMARK 3.9. We must point out that if $\varphi$ and $\psi$ are two point supports in $X$ and $Y$ respectively and $f: X \rightarrow Y$ is a proper map (w.r.t. $\varphi$ and $\psi$ ) which is a local homeomorphism, then $H_{\varphi}^{p}(X) \approx H_{\psi}^{p}(Y)$, for every $p \geq 0$. Also we observe that if $U$ is any open neighbourhood of the point defining $\varphi$ and $V$ is any open neighbourhood of the point defining $\psi$, then clearly $H_{\varphi}^{p}(X) \approx H_{\varphi}^{p}(U)$ and $H_{\psi}^{p}(Y) \approx H_{\psi}^{p}(V)$. Therefore sheaf cohomology with point supports can be quite useful in deciding that two spaces $X$ and $Y$ are not locally homeomorphic. We shall illustrate this by means of some examples.

EXAMPLE 3.10. A very often quoted example of two spaces $X$ and $Y$ which have isomorphic homology and cohomology groups in all dimensions and for all coefficients, but do not have isomorphic cup products is the following ([6], page 253): $X=S^{p} \times S^{q}, p<q$ say, and $Y=S^{p} \vee S^{q} \vee S^{p+q}$. Here $S^{p}, S^{q}$ etc. are spheres of dimension $p$ and $q$ respectively and $Y$ is the one point union of $S^{p}, S^{q}$ and $S^{p+q}$. The main point of this example is to show that $X, Y$ can not be distinguished only by the additive structures of homology and cohomology groups; 
it is only the multiplicative structure of cohomology which can distinguish them. However, the concept of cohomology with point supports can be more precise than cohomology with supports in cld. We now show that only the additive structure of cohomology with point supports can distinguish between the above spaces $X$ and $Y$. Let $\varphi$ be the point support in $X$ defined by any point of $X$. Then for each open neighbourhood $U$ of that point

$$
H_{\varphi}^{n}(U) \approx \begin{cases}\mathbb{Z} & \text { if } n=p, q \text { or } p+q \\ 0 & \text { otherwise. }\end{cases}
$$

But if $\psi$ is the point support in $Y$ defined by the distinguished point, then one can easily see that for any open neighbourhood $V$ of that point

$$
H_{\psi}^{n}(V) \approx \begin{cases}\mathbb{Z} \oplus \mathbb{Z} \oplus \mathbb{Z} & \text { if } n=1, \\ \mathbb{Z} & \text { if } n=p, q \text { or } p+q, \\ 0 & \text { otherwise. }\end{cases}
$$

If $\psi$ is the point support defined by any other point of $Y$ and $V$ is any open neighbourhood of that point, then we can compute

$$
H_{\psi}^{n}(V) \approx \begin{cases}\mathbb{Z} & \text { if } n=p \text { or } q \text { or } p+q \\ 0 & \text { otherwise. }\end{cases}
$$

These groups show, as pointed out in the above remark, that $X$ and $Y$ cannot be locally homeomorphic even at one point.

EXAMPLE 3.11. There is yet another famous example of two spaces $X$ and $Y$ ([6], page 275) where $X=S^{3} \vee S^{5}$ and $Y=C P^{2} \times I$ which cannot be distinguished even by their cohomology ring structures in the sense that both have not only isomorphic homology and cohomology groups but also have isomorphic cup and cap products, they can be distinguished by only higher order Steenrod operations. Just as in Example 3.10, we can easily show that these spaces can also be distinguished by only the additive structure of the point support cohomology; in fact, if we take $\varphi$ to be the point support of $X$ containing only the distinguished point, then $H_{\varphi}^{1}(X) \approx \mathbb{Z}$ whereas $H_{\psi}^{1}(Y)=0$ for any point support $\psi$ in $Y$.

On large cohomology dimension. Recall that the large cohomology dimension $\operatorname{Dim}_{\mathbf{Z}}(X)$ of a space $X$ is the largest integer $n$ (or $\infty$ ) for which there exists a sheaf $\mathscr{A}$ of abelian groups on $X$ and a family of supports $\varphi$ on $X$ such that $H_{\varphi}^{n}(X ; \mathscr{A}) \neq 0$. It was proved in [3] that $\operatorname{Dim}_{\mathbf{Z}}(\mathbf{R})=2$, and using this result it was proved in [4] that if $X$ is an $n$-manifold then $\operatorname{Dim}_{\mathbf{Z}}(X)=n+1$. In the proofs, sheaves were explicitly constructed, but the family of supports $\varphi$ was only shown to exist, it was not explicitly constructed. Now we are going to use the concept of point supports to give a direct proof of this result by explicitly 
constructing both the sheaf and the family $\varphi$ of supports. It is interesting to note that the family $\varphi$ consisting of a point support does the job! We have

THEOREM 3.12 (DEO). If $X$ is an $n$-manifold, then $\operatorname{Dim}_{\mathbf{Z}}(X)=n+1$.

PROOF. It is obviously sufficient to prove the result for $X=\mathbf{R}^{n}$. Let $B(1 / m)$ denote the closed ball of radius $1 / m$ around origin and suppose $B^{\circ}(1 / m)$ denotes the corresponding open ball. Let $A_{m}=B(1 / m)-B^{\circ}(1 /(m+1))$. Then $\left\{A_{m} \mid m=1,4,7,9, \ldots\right\}$ is a disjoint family of closed subsets of $X$. If we put $W=\bigcup\left\{A_{m} \mid m=1,4,7,9, \ldots\right\}$, and $F=W \cup\{0\}$ where 0 is the origin, then $W$ is open in $F$. Note that each $A_{m}$ is of the same homotopy type as $(n-1)$-sphere. Hence it is easy to compute

$$
H_{\mathrm{cld}}^{p}(W) \approx \begin{cases}\prod_{i \in \mathrm{N}} \mathbf{Z}_{i} & \text { if } p=n-1 \text { or } 0 \\ 0 & \text { otherwise }\end{cases}
$$

and

$$
H_{\text {cld }}^{p}(F) \approx \begin{cases}\bigoplus_{i \in \mathrm{N}} \mathbf{Z}_{i} & \text { if } p=n-1 \text { or } 0, \\ 0 & \text { otherwise }\end{cases}
$$

where $\mathbf{Z}_{i} \approx \mathbf{Z}$, for every $i$. Now the following part of the exact sequence of the pair $(F, W)$ (supports in cld)

$$
\cdots \rightarrow H^{n-1}(F, W) \rightarrow H^{n-1}(F) \rightarrow H^{n-1}(W) \rightarrow H^{n}(F, W) \rightarrow \cdots
$$

shows that $H^{n}(F, W)$ cannot be finitely generated. Since $W$ is open in $F$, it is taut and we find that ([1], page 59) $H^{n}(F, W) \approx H_{\varphi}^{n}(F)$ where $\varphi=\operatorname{cld} \mid F-W$ is the point support. Once again let us consider the following part of the exact sequence of the pair $\left(\mathbf{R}^{n}, F\right)$ with supports in $\varphi$.

$$
\cdots \rightarrow H_{\varphi}^{n}\left(\mathbf{R}^{n}, F\right) \rightarrow H_{\varphi}^{n}\left(\mathbf{R}^{n}\right) \rightarrow H_{\varphi}^{n}(F) \rightarrow H_{\varphi}^{n+1}\left(\mathbf{R}^{n}, F\right) \rightarrow \cdots .
$$

Since $H_{\varphi}^{n}\left(\mathbf{R}^{n}\right) \approx \mathbf{Z}$ and $H_{\varphi}^{n}(F)$ is not finitely generated, $H_{\varphi}^{n+1}\left(\mathbf{R}^{n}, F\right) \neq 0$. Since $F$ is closed in $\mathbf{R}^{n}$, we find ([1], page 59 ) that

$$
0 \neq H_{\varphi}^{n+1}\left(\boldsymbol{R}^{n}, F\right) \approx H_{\varphi}^{n+1}\left(\boldsymbol{R}^{n}, \mathbf{Z}_{\boldsymbol{R}^{n}-F}\right) .
$$

This proves that $\operatorname{Dim}_{\mathbf{Z}}\left(\mathbf{R}^{n}\right)=n+1$.

\section{Some examples}

Next we give some examples to show that our Theorem 2.1 is quite sharp. In this context first let us recall the following results proved in ([1], page 68). 
Proposition 4.1. Let $X=X_{1} \cup X_{2}$ and $A=X_{1} \cap X_{2}$. If $\varphi$ is a family of supports on $X$ such that

(A) $\left(\varphi \cap X_{1}\right)\left|X_{1}-A=\varphi\right| X-X_{2} \subset \varphi \mid \operatorname{Int}\left(X_{1}\right)$, and

(B) $X_{2}$ is $\varphi$-taut in $X, A$ is $\left(\varphi \cap X_{1}\right)$-taut in $X_{1}$, then the pair $\left(X_{1}, X_{2}\right)$ is $\varphi$-excisive.

COROLlaRY 4.2. If $X=\operatorname{Int}\left(X_{1}\right) \cup X_{2}=X_{1} \cup \operatorname{Int}\left(X_{2}\right)$ and $X_{1}, X_{2}, X_{1} \cap X_{2}$ are all $\varphi$-taut in $X$, then $\left(X_{1}, X_{2}\right)$ is $\varphi$-excisive.

As pointed out in the introduction it has been an open question whether or not the tautness conditions in the above proposition and corollary are really needed. In [2] an example has been constructed in which all conditions of Proposition 4.1 except (B) are satisfied and the pair $\left(X_{1}, X_{2}\right)$ is not $\varphi$-excisive. This showed that the tautness conditions in the Proposition 4.1 are required and the condition (A) alone is not enough for the pair $\left(X_{1}, X_{2}\right)$ to be $\varphi$-excisive. Note, however, that the condition in the Corollary 4.2 corresponding to the condition (A) of the Proposition 4.1 is stronger and our Theorem 2.1 shows that tautness conditions in the Corollary are not needed. We are going now to produce an example which will show that nothing weaker than the hypothesis of Theorem 2.1 can imply that the pair $\left(X_{1}, X_{2}\right)$ is $\varphi$-excisive. In fact in our example we will have $X=\operatorname{Int}\left(X_{1}\right) \cup X_{2}$ and $X_{1}, X_{1} \cap X_{2}, X_{2}$ all $\varphi$-taut in $X$, even then the pair $\left(X_{1}, X_{2}\right)$ is not $\varphi$-excisive. We have

EXAMPLE 4.3. Let $X=\mathbf{R}^{2}$ and $\varphi=\operatorname{cld} \mid X-D^{2}$ where $D^{2}=\{(x, y) \in$ $\left.X \mid x^{2}+y^{2} \leq 1\right\}$. Let $X_{1}=\left\{(x, y) \in X \mid y \leq 0\right.$ and $\left.x^{2}+y^{2}>2\right\} \cup\{(-2,0),(2,0)\}$, and $X_{2}=\left\{(x, y) \in X \mid y \geq 0\right.$ or $\left.x^{2}+y^{2} \leq 2\right\}$. Note that $\varphi$ is a paracompactifying family of supports and since for such a family any subset of a completely paracompact space is $\varphi$-taut, $X_{1}, X_{2}, X_{1} \cap X_{2}$ are all $\varphi$-taut in $X$. Next we compute the cohomology groups of the constant sheaf $Z$ on $X_{1}, X_{2}, X_{1} \cap X_{2}, X$ with supports in $\varphi \cap X_{1}, \varphi \cap X_{2}, \varphi \cap\left(X_{1} \cap X_{2}\right), \varphi$ respectively (which we indicate only by $\varphi$ ). Since $X$ is $\varphi$-contractible to any point of $\operatorname{Int}\left(D^{2}\right), H_{\varphi}^{*}(X)=0$. Similarly because $X_{2}$ is $\varphi$-contractible to any point of $\operatorname{Int}\left(D^{2}\right), H_{\varphi}^{*}\left(X_{2}\right)=0$. However, $X_{1} \in \varphi$ and since $X_{1}$ is connected and contractible we get

$$
H_{\varphi}^{0}\left(X_{1}\right) \approx \mathbb{Z} \text { and } H_{\varphi}^{p}\left(X_{1}\right)=0, \quad \text { for every } p>0 .
$$

Also $X_{1} \cap X_{2}$ has two connected components (each $\varphi$-contractible) and both are members of $\varphi$, therefore

$$
H_{\varphi}^{0}\left(X_{1} \cap X_{2}\right) \approx \mathbf{Z} \oplus \mathbb{Z} \text { and } H_{\varphi}^{p}\left(X_{1} \cap X_{2}\right)=0 \text {, for every } p>0 .
$$

Thus the following part of the Mayer-Vietoris sequence for the pair $\left(X_{1}, X_{2}\right)$

$$
0 \rightarrow H_{\varphi}^{0}(X) \rightarrow H_{\varphi}^{0}\left(X_{1}\right) \oplus H_{\varphi}^{0}\left(X_{2}\right) \rightarrow H_{\varphi}^{0}\left(X_{1} \cap X_{2}\right) \rightarrow H_{\varphi}^{1}(X) \rightarrow \cdots
$$


takes the form $0 \rightarrow \mathbb{Z} \rightarrow \mathbb{Z} \oplus \mathbb{Z} \rightarrow 0$, which cannote be exact. Thus $\left(X_{1}, X_{2}\right)$ cannot be $\varphi$-excisive.

This example shows that just as in the presence of condition (A), the condition (B) in the Proposition 4.1 is needed, similarly in the presence of condition (B) the condition (A) of the Proposition 4.1 is also needed, that is, both the conditions of Proposition 4.1 are separately required.

Next we use the results of Section 3 to give an example to illustrate our Theorem 2.1. We will give subspaces $X_{1}, X_{2}$ of a space $X$ and a family $\varphi$ of supports on $X$ such that $X=\operatorname{Int}\left(X_{1}\right) \cup X_{2}=X_{1} \cup \operatorname{Int}\left(X_{2}\right)$ and the pair $\left(X_{1}, X_{2}\right)$ is $\varphi$-excisive although none of the space $X_{1}, X_{2}, X_{1} \cap X_{2}$ is $\varphi$-taut in $X$. Also this example is not covered under any of the known Theorem (1.1), (1.2) or (1.3).

EXAMPLE 4.4. Let $X=\mathbf{R}^{2}$ and $\varphi$ be the family of suports consisting of only the origin of $\mathbf{R}^{2}$. Let

$$
\begin{aligned}
& X_{1}=\{(x, y) \in X \mid y>0 \text { or } y<0\} \cup\{(0,0)\}, \text { and } \\
& X_{2}=\{(x, y) \in X \mid x<0 \text { or } x>0\} \cup\{(0,0)\} .
\end{aligned}
$$

Then it is clear that $X=\operatorname{Int}\left(X_{1}\right) \cup X_{2}=X_{1} \cup \operatorname{Int}\left(X_{2}\right)$. Now we shall verify that the Mayer-Vietoris sequence for the pair $\left(X_{1}, X_{2}\right)$ for the constant sheaf $\mathbb{Z}$ of integers and with supports in $\varphi$ is exact and will show that none of the spaces $X_{1}, X_{2}, X_{1} \cap X_{2}$ is $\varphi$-taut in $X$.

To compute the cohomology groups of $X_{1}$ with supports in $\varphi \cap X_{1}$ (which we indicate only by $\varphi$ ), let us consider the following closed subsets of $X_{1}$,

$$
\begin{aligned}
& K_{1}=\{(x, y) \in X \mid y>0\} \cup\{(0,0)\}, \text { and } \\
& K_{2}=\{(x, y) \in X \mid y<0\} \cup\{(0,0)\} .
\end{aligned}
$$

It is easy to see that $K_{1}$ and $K_{2}$ are $\varphi$-contractible to suitable points and hence $H_{\varphi}^{*}\left(K_{1}\right)=0=H_{\varphi}^{*}\left(K_{2}\right)$. Since $K_{1}, K_{2}$ are closed in $X_{1}$, we can apply the exact Mayer-Vietoris sequence to get that

$$
H_{\varphi}^{p}\left(X_{1}\right) \approx \begin{cases}\mathbb{Z} & \text { if } p=1 \\ 0 & \text { otherwise }\end{cases}
$$

Similarly, we obtain

$$
H_{\varphi}^{p}\left(X_{2}\right) \approx \begin{cases}\mathbb{Z} & \text { if } p=1 \\ 0 & \text { otherwise. }\end{cases}
$$

By Proposition 3.5, we have

$$
H_{\varphi}^{p}(X) \approx \begin{cases}\mathbb{Z} & \text { if } p=2, \\ 0 & \text { otherwise. }\end{cases}
$$

Now $X_{1} \cap X_{2}=Q_{1} \cup Q_{2} \cup Q_{3} \cup Q_{4} \cup\{(0,0)\}$ where $Q_{1}, Q_{2}, Q_{3}, Q_{4}$ are the first, second, third and fourth open quadrants of $\mathbf{R}^{2}$. Since $Q_{1} \cup\{(0,0)\}, Q_{2} \cup\{(0,0)\}$ 
are closed in $Q_{1} \cup Q_{2} \cup\{(0,0)\}$ and each is $\varphi$-acyclic, we can apply the MayerVietoris sequence repeatedly to find that

$$
H_{\varphi}^{p}\left(X_{1} \cap X_{2}\right) \approx \begin{cases}\mathbb{Z} \oplus \mathbb{Z} \oplus \mathbb{Z} & \text { if } p=1, \\ 0 & \text { otherwise. }\end{cases}
$$

Thus the Mayer-Vietoris sequence for the pair $\left(X_{1}, X_{2}\right)$, viz.,

$$
\begin{gathered}
\ldots \rightarrow H_{\varphi}^{0}\left(X_{1} \cap X_{2}\right) \rightarrow H_{\varphi}^{1}(X) \rightarrow H_{\varphi}^{1}\left(X_{1}\right) \oplus H_{\varphi}^{1}\left(X_{2}\right) \rightarrow H_{\varphi}^{1}\left(X_{1} \cap X_{2}\right) \\
\rightarrow H_{\varphi}^{2}(X) \rightarrow H_{\varphi}^{2}\left(X_{1}\right) \oplus H_{\varphi}^{2}\left(X_{2}\right) \rightarrow \cdots
\end{gathered}
$$

takes the form

which is exact.

$$
0 \rightarrow \mathbb{Z} \oplus \mathbb{Z} \rightarrow \mathbb{Z} \oplus \mathbb{Z} \oplus \mathbb{Z} \rightarrow \mathbb{Z} \rightarrow 0,
$$

Next we show that $X_{1}$ is not $\varphi$-taut in $X$. For, any neighbourhood $U$ of $X_{1}$ in $X$ is obviously a neighbourhood of origin, and hence by Proposition 3.8, $H_{\varphi}^{2}(U) \approx \mathbb{Z}$. Also if $V$ is any other neighbourhood of $X_{1}$ such that $V \subseteq U$, then $H_{\varphi}^{2}(V) \approx \mathbb{Z}$ and the restriction homomorphism $H_{\varphi}^{2}(U) \rightarrow H_{\varphi}^{2}(V)$ is an isomorphism. Hence $\lim _{\varphi} H_{\varphi}^{2}(U) \approx \mathbb{Z}$, where $U$ ranges over all neighbourhoods of $X_{1}$ in $X$. But $H_{\varphi}^{2}\left(\overrightarrow{\left.X_{1}\right)}=0\right.$, which shows that $X_{1}$ cannot be $\varphi$-taut in $X$. By similar arguments one can easily verify that neither $X_{2}$ nor $X_{1} \cap X_{2}$ is $\varphi$-taut in $X$.

Next suppose we take the same spaces $X, X_{1}$ and $X_{2}$ and a different exotic family of supports $\varphi$ described as follows: Let $\varphi$ be the smallest family of supports containing cld $\mid Q_{i}(i=1,2,3,4)$ where $Q_{i}$ are open quadrants of $\mathbf{R}^{2}$ and any straight line passing through origin (other than the coordinate axes). Then we can compute using Proposition 3.8

$$
H_{\varphi}^{p}\left(X_{1}\right) \approx \begin{cases}5 & \text { if } p=1 \\ 0 & \text { otherwise }\end{cases}
$$

Here and afterwards 5 means five copies of $\mathbf{Z}$.

$$
\begin{aligned}
H_{\varphi}^{p}\left(X_{2}\right) & \approx \begin{cases}5 & \text { if } p=1, \\
0 & \text { otherwise },\end{cases} \\
H_{\varphi}^{p}\left(X_{1} \cap X_{2}\right) & \approx \begin{cases}7 & \text { if } p=1, \\
0 & \text { otherwise }\end{cases}
\end{aligned}
$$

Also $H_{\varphi}^{p}(X)$ is given by the following exact sequence

$$
0 \rightarrow H_{\varphi}^{1}(X) \rightarrow 4 \rightarrow 1 \rightarrow H_{\varphi}^{2}(X) \rightarrow 0 .
$$

If $H_{\varphi}^{2}(X)=0$, then $H_{\varphi}^{1}(X)=3$ and if $H_{\varphi}^{2}(X)=1$, then $H_{\varphi}^{1}(X)=4$.

In any case the Mayer-Vietoris sequence for the pair $\left(X_{1}, X_{2}\right)$ takes the form

$$
\begin{gathered}
0 \rightarrow 3 \rightarrow 10 \rightarrow 7 \rightarrow 0 \\
\text { or } \\
0 \rightarrow 4 \rightarrow 10 \rightarrow 7 \rightarrow 1 \rightarrow 0,
\end{gathered}
$$

and these are exact. 
Finally let us take $\varphi$ to be the smallest family of supports containing cld $\mid Q_{i}$ $(i=1,2,3,4)$ and origin. Then again we can compute using Proposition 3.8 and Example 3.4(b)

$$
\begin{gathered}
H_{\varphi}^{p}\left(X_{1}\right) \approx \begin{cases}1 & \text { if } p=1, \\
4 & \text { if } p=2, \\
0 & \text { otherwise }\end{cases} \\
H_{\varphi}^{p}\left(X_{2}\right) \approx \begin{cases}1 & \text { if } p=1, \\
4 & \text { if } p=2, \\
0 & \text { otherwise }\end{cases} \\
H_{\varphi}^{p}\left(X_{1} \cap X_{2}\right) \approx \begin{cases}3 & \text { if } p=1, \\
4 & \text { if } p=2, \\
0 & \text { otherwise }\end{cases} \\
H_{\varphi}^{p}(X) \approx \begin{cases}5 & \text { if } p=2, \\
0 & \text { otherwise }\end{cases}
\end{gathered}
$$

and the nontrivial part of the Mayer-Vietoris sequence for the pair $\left(X_{1}, X_{2}\right)$ takes the form

$$
0 \rightarrow 2 \rightarrow 3 \rightarrow 5 \rightarrow 8 \rightarrow 4 \rightarrow 0
$$

which is exact.

\section{References}

[1] G. E. Bredon, Sheaf theory (McGraw-Hill, New York, 1967).

[2] S. Deo, 'An example of nonexcisiveness in sheaf cohomology', Proc. Amer. Math. Soc. 17 (1975), 501-503.

[3] S. Deo, 'One dimensional manifold is of cohomological dimension two', Proc. Amer. Math. Soc. 52 (1975), 445-446.

[4] S. Deo, 'The cohomological dimension of an $n$-manifold is $n+1$ ', Pacific J. Math. 67 (1976), 154-160.

[5] R. Godement, Topologie algébrique et théorie des faisceaux (Hermann, Paris, 1958).

[6] E. H. Spanier, Algebraic topology (McGraw-Hill, New York, 1966).

Department of Mathematics

University of Jammu

Jammu-180 001

India 